\title{
Generalization of the Variational Principle and the Hohenberg and Kohn Theorems for Excited States of Fermion Systems
}

\author{
A. Gonis \\ Physical and Life Sciences, Lawrence Livermore National Laboratory, PO Box 808, L-45, \\ Livermore, CA 94551
}

\begin{abstract}
Through the entanglement of a collection of $K$ non-interacting replicas of a system of $N$ interacting Fermions, and making use of the properties of reduced density matrices the variational principle and the theorems of Hohenberg and Kohn are generalized to excited states. The generalization of the variational principle makes use of the natural orbitals of an $N$-particle density matrix describing the state of lowest energy of the entangled state. The extension of the theorems of Hohenberg and Kohn is based on the ground-state formulation of density functional theory but with a new interpretation of the concept of a ground state: It is the state of lowest energy of a system of $K N$ Fermions that is described in terms of the excited states of the $N$-particle interacting system. This straightforward implementation of the line of reasoning of ground-state density functional theory to a new domain leads to a unique and logically valid extension of the theory to excited states that allows the systematic treatment of all states in the spectrum of the Hamiltonian of an interacting system.
\end{abstract}

Keywords: excited states, density functional theory, variational theorem, Hohenberg and Kohn Theorems

2010 MSC: 00-01, 99-00

\footnotetext{
${ }^{\star 2}$ Fully documented templates are available in the elsarticle package on CTAN

${ }^{1}$ Since 1880 .
}

Preprint submitted to Physics Letters A

August 16, 2016

(C) 2016. This manuscript version is made available under the Elsevier user license http://www.elsevier.com/open-access/userlicense/1.0/ 


\section{Introduction}

The extension to excited states of density functional theory, introduced by Hohenberg and Kohn [1] with respect to the ground states of interacting, many particle systems, has preoccupied quantum chemists and condensed mat-

5 ter physicists for decades. Unfortunately, however, in spite of a great number of attempts in that direction (of which only a representative sample is cited here [2, 3, 4, 5, 6, 7, 8, 9, 10, 11, 12, 13, 14, 15, 16, 17, 18, 19, 20, 21, 22, 23, 24, [25, 26, 27, 28, 29, 30]), the task has not been accomplished.

This is not for want of ingenuity or intensity of effort, for there has been much along both directions. It is to say that these efforts have come in many forms and varied lines of reasoning to lack both coherence and uniformity, and hence convincing power. Above all, they fail to satisfy the one principle that governs the extension of a theoretical construct in mathematical physics to a realm broader than that of its prior implementation. Namely, that the extension retains intact the initial language describing the method, with the extension materializing through the application of that language to elements outside the domain of previous usage.

Other conceptual difficulties are also present. Many, if not all, of these forms seem to subscribe to the notion that density functional theory is a theory of functionals of the density that are mere functions of the density, geared at the determination of ground-state energies as its ultimate goal. On the contrary, as suggested by this work, density functional theory is a body of work in mathematical physics that provides an alternative to the Schrödinger equation in determining the quantum states of interacting many-particle systems, ground as well as excited. If this general theory is to be expressed in the language of its ground-state formulation, then, at least in the static limit and in the absence of vector potentials and fields, it must satisfy the requirement that all developments within the realm of density functional theory must spring from a single density, and only that density, that potentially describes the ground state of an interacting many-particle system. It follows that to study excited states within 
this theory, it is necessary to devise an interacting system of particles whose ground state can be described in terms of the excited states of an interacting $N$ particle system. Here, I develop this theory for the case of Fermions.

As is shown below, such a system consists of the entanglement into a single 35 system of a number, $K$, of mutually non-interacting replicas of a given interacting system of $N$ Fermions (e.g., electrons), described by an antisymmetric wave function of $K N$ particles governed by a Hamiltonian of an interacting $N$ particle system. The Hamiltonian acts on all possible sets of $N$-particles without coupling any one of the sets to any other. Consistent with the quantum requirement that physical systems of Fermions be represented by anti-symmetric wave functions (change sign under interchange of particle coordinates, (and spins, for electrons)), the entanglement into one state obeying the Pauli exclusion principle obliterates the distinguishability of particles in different replicas whose individual states are no longer discernible in the entangled state. It turns out

45 that the ground state of this system, corresponding to the lowest energy state of the replica collection, i.e., the single entangled pure state, yields $C_{N}^{K N}=\left(\begin{array}{c}K N \\ N\end{array}\right)$ states of the interacting $N$ particle system. The full spectrum is obtained in the limit, $K \rightarrow \infty$. The aim is to describe these states within the formalism of ground-state DFT.

50 As in the case of the ground-state formulation of DFT, its extension to excited states is rooted in the corresponding extension of the variational principle.

\section{Variational Principle for Excited States}

For simplicity of exposition in the following, I suppress spin unless noted otherwise. Also because I wish to follow as closely as possible the steps in the original paper of Hohenberg and Kohn, I will suppress the presence of degeneracy.

In addition, I consider explicitly the case of electrons, although without spin, and all wave functions are assumed to have unit normalization. In what follows, the terms particles and electrons are used interchangeably. 
In its conventional formulation, the variational principle is used to determine the ground state of an interacting $N$-electron system by identifying that antisymmetric $N$-particle wave function that minimizes the expectation value of the Hamiltonian,

$$
\hat{H}^{N}=\hat{v}^{N}+\hat{T}^{N}+\hat{U}^{N},
$$

${ }_{60}$ where $\hat{T}^{N}$ and $\hat{U}^{N}$ are the kinetic and inter-particle interaction (Coulomb interaction for electrons) operators for the system, and $\hat{v}^{N}$ is the operator representing an external potential, $v^{N}(\mathbf{r})$, acting on a particle at $\mathbf{r}$.

Let $\mathcal{I}_{L}$ be the set of all anti-symmetric wave functions of $L$ particles, with unit normalization. Then the state of lowest energy, ground state, of a collection of $K$ replicas of an interacting system of $N$ electrons, for a total of $K N$ particles, is obtained from the minimization procedure,

$$
\begin{aligned}
E= & \underbrace{\operatorname{Min}}_{|\Psi\rangle \in \mathcal{I}_{K N}}\left\langle\Psi^{K N}\left|\hat{H}^{N}\right| \Psi^{K N}\right\rangle \\
& =\left\langle\Psi_{\mathrm{GS}}^{K N}\left|\hat{H}^{N}\right| \Psi_{\mathrm{GS}}^{K N}\right\rangle .
\end{aligned}
$$

The minimizing wave function is denoted by $\left|\Psi_{\mathrm{GS}}^{K N}\right\rangle$ and the quantity, $E$, is the ground-state energy of the replica system.

Let $\mathbf{r}_{(N)}=\left(\mathbf{r}_{1}, \mathbf{r}_{2}, \ldots, \mathbf{r}_{N}\right)$.

From [31, define the $N$ th order reduced density matrix,

$$
\begin{aligned}
& \hat{\Gamma}_{N}\left(\mathbf{r}_{(N)}^{\prime} ; \mathbf{r}_{(N)}\right)=C_{N}^{K N} \int \Psi_{\mathrm{GS}}^{K N *}\left(\mathbf{r}_{(N)}^{\prime}, \mathbf{r}_{N+1}, \ldots, \mathbf{r}_{K N}\right) \\
\times \quad & \Psi_{\mathrm{GS}}^{K N}\left(\mathbf{r}_{(N)}, \mathbf{r}_{N+1}, \ldots, \mathbf{r}_{K N}\right) \mathrm{d} \mathbf{r}_{N+1} \ldots \mathrm{d} \mathbf{r}_{K N},
\end{aligned}
$$

which can also be written in terms of its eigenstates and corresponding eigenvalues,

$$
\hat{\Gamma}_{N}\left(\mathbf{r}_{(N)}^{\prime} ; \mathbf{r}_{(N)}\right)=\sum_{J=1} \lambda_{J} D_{J}^{N}\left(\mathbf{r}_{(N)}^{\prime}\right) D_{J}^{N}\left(\mathbf{r}_{(N)}\right),
$$

with the $\left|D_{J}^{N}\right\rangle$ forming a mutually orthonormal set,

$$
\left\langle D_{J}^{N} \mid D_{I}^{N}\right\rangle=\delta_{I J} .
$$

From (4) and (5) follows that,

$$
\operatorname{Tr} \hat{\Gamma}_{N}=\sum_{J} \lambda_{J}=C_{N}^{K N} .
$$


The $\left|D_{J}^{K N}\right\rangle$ can be used to express the full anti-symmetric wave function, $\Psi_{\mathrm{GS}}^{K N}\left(\mathbf{r}_{(K N)}\right)$, in the form [31],

$$
\begin{aligned}
& \Psi_{\mathrm{GS}}^{K N}\left(\mathbf{r}_{(K N)}\right)=\frac{1}{\sqrt{C_{N}^{K N}}} \\
\times & \sum_{J=1}^{C_{N}^{K N}} D_{J}^{N}\left(\mathbf{r}_{(N)}\right) M_{J}^{(K-1) N}\left(\mathbf{r}_{N+1}, \ldots, \mathbf{r}_{K N}\right),
\end{aligned}
$$

where the wave functions $M_{J}^{(K-1) N}\left(\mathbf{r}_{((K-1) N)}\right)$ are mutually orthonormal, are orthogonal to the corresponding $D_{J}^{N}\left(\mathbf{r}_{(N)}\right)$ and have the same eigenvalues [31]. Now, a density normalized to $N$ but representing the ground state of the replica system is obtained in the form,

$$
n\left(\mathbf{r}_{1}\right)=N \int\left|\Psi_{\mathrm{GS}}^{K N}\left(\mathbf{r}_{1}, \mathbf{r}_{2}, \ldots, \mathbf{r}_{K N}\right)\right|^{2} \mathrm{~d} \mathbf{r}_{2} \ldots \mathrm{d} \mathbf{r}_{K N} .
$$

\section{Ground State Energy}

The expression (8) leads to the expression,

$$
E=\frac{1}{C_{N}^{K N}} \sum_{J=1}\left\langle D_{J}^{N}\left|\hat{H}^{N}\right| D_{J}^{N}\right\rangle
$$

where because of the orthonormality of the $\left|D_{J}^{N}\right\rangle$, the expectation values can be

ordered from smaller to larger. Assume this ordering has been effected in the last expression, with each state maximally occupied. It remains to determine these states.

Expand each of the $\left|D_{J}^{N}\right\rangle$ in terms of the eigenstates, $\left|\Psi_{L}^{N}\right\rangle$, of $\hat{H}^{N}$,

$$
\hat{H}^{N}\left|\Psi_{L}\right\rangle=E_{L}\left|\Psi_{L}^{N}\right\rangle
$$

obtaining,

$$
\left|D_{J}^{N}\right\rangle=\sum_{J} C_{L}^{J}\left|\Psi_{L}^{N}\right\rangle
$$

so that, due to the orthogonality of the eigenstates, $\left|\Psi_{L}^{N}\right\rangle$,

$$
E=\frac{1}{C_{N}^{K N}} \sum_{J=1} \sum_{L}\left|C_{L}^{J}\right|^{2} E_{L}
$$


The lowest value of the $J=1$ term in $10 p$ is $E_{1}$, the ground-state energy of $\hat{H}^{N}$. Occupying each successive level with the lowest in value eigenenergy of the Hamiltonian that is the next higher compared to the previous level, we find,

$$
E=\frac{1}{C_{N}^{K N}} \sum_{J=1}^{C_{N}^{K N}} E_{J},
$$

where the $C_{N}^{K N}$ excitation energies, $E_{J}$, are arranged in ascending order. However, these energies are not sequentially the lowest in energy. Indeed, the variational principle in this case leads to a minimizing wave function that can be written in terms of those excited states of $\hat{H}^{N}$ that are simultaneously eigenstates of $\hat{\Gamma}_{N}$. Because there are $N$ particles in the ground state that can be exchanged with $(K-1) N$ particles in excited states, there are $C_{1}^{N} C_{1}^{(K-1) N}$ single-particle excitations. Similarly, there are $C_{2}^{N} C_{2}^{(K-1) N}$ two-particle excitations, and so on, to $C_{N}^{N} C_{N}^{(K-1) N}$ excitations of order $N$. It is easily verified,

$$
\sum_{m=0}^{N} C_{m}^{N} C_{m}^{(K-1) N}=C_{N}^{K N},
$$

the total number of excited states. The gaps generated in the spectrum are seen easily: After the highest single-particle excitation, the energies jump to the lowest in energy two-particle excitation, leaving a gap to be filled by the remaining single-particle excitations (an infinite number). Similarly there is a gap between the highest in energy two-particle excitation and the lowest in energy three-particle one. And so on to the highest in energy $N$-particle excited state.

Increasing the number of replicas, $K$, the lower level excitations are filled in progressively more and more densely, while new gaps are opening up at higher energies. The entire spectrum is generated as $K \rightarrow \infty$.

For example, for $N=2$ and $K=2$, say spineless He, there are 4 electrons and $C_{2}^{4}=6$ states, the ground state, 4 single-particle excitations, and one twoparticle excitation. $K=3$, leads to 1 ground state, 8 single particle excitations and 6 two-particle excitations, for a total of $C_{2}^{6}=15$ states. 
From this follows that in the case of the minimizing wave function, $\Psi_{\mathrm{GS}}^{K N}\left(\mathbf{r}_{(K N)}\right)$, the eigenfunctions of the $\mathrm{N}$-particle density matrix correspond to those eigenstates of $\hat{H}^{N}$ that form an antisymmetric wave function in the form of (8). 95 A formal methodology for explicitly determining the excited states, i.e., the eigenstates of the $N$ th order density matrix corresponding to the ground state, $\left|\Psi^{K N}\right\rangle$ will be discussed in future work.

It now follows that in the absence of degeneracy the $N$ lowest in energy eigenstates (orbitals or spin orbitals) of a single-particle Hamiltonian, $\hat{H}^{1}=$ $\hat{v}+\hat{T}^{1}$, are given by a Slater determinant that is the ground state of a noninteracting $N$ particle system under the action of $\hat{v}$. The result that the nondegenerate ground state of $N$ non-interacting particles under a potential, $\mathrm{v}(\mathbf{r})$, is a Slater determinant has also been established by Lieb [37] following a different line of reasoning.

Finally, the following result is central to the extension of DFT to excited states.

Let $\Psi_{1}^{K N}$ and $\Psi_{2}^{K N}$ minimize the expectation values of $\hat{H}_{1}^{N}$ and $\hat{H}_{2}^{N}$, determined in terms of potentials, $v_{1}$ and $v_{2}$, respectively, that differ by more than a constant, $v_{1} \neq v_{2}+c$. With $E_{1}$ the ground state energy of $K$ replicas of the system $\hat{H}_{1}^{N}$, we have,

$$
E_{1}<\left\langle\Psi_{2}^{K N}\left|\hat{H}_{1}^{N}\right| \Psi_{2}^{K N}\right\rangle,
$$

with a corresponding inequality with the indices 1 and 2 interchanged.

At this point, an observation is in order. The Hohenberg and Kohn theorems apply to particles of arbitrary symmetry including distinguishable particles.

Having demonstrated the generalization of the variational principle to excited states that is not limited by symmetry (applies to both Fermions and Bosons, as well as distinguishable particles) the proof could very well end here. However, the following section, where the proof is repeated, is intended to strengthen the complete and direct analogy between the strictly ground state case and that of excited states treated within the formalism. 


\section{Generalization of the Hohenberg and Kohn Theorems}

The expectation value of $\sum_{j=1}^{N} v\left(\mathbf{r}_{i}\right)$ with respect to $\Psi_{\mathrm{GS}}^{K N}\left(\mathbf{r}_{(K N)}\right)$ gives,

$$
\left\langle\Psi_{\mathrm{GS}}^{K N}\left|\sum_{j=1}^{N} v\left(\mathbf{r}_{i}\right)\right| \Psi_{\mathrm{GS}}^{K N}\right\rangle=\int v(\mathbf{r}) n(\mathbf{r}) \mathrm{d} \mathbf{r},
$$

with the density normalized to $N$, Eq. (9).

It is now shown that it is not possible for two wave functions to lead to the same density and correspond to the ground state of two different potentials that differ by more than a constant. Letting $\Psi_{1}^{K N}$ and $\Psi_{2}^{K N}$ lead to the same density, $n(\mathbf{r})$, and minimize the expectation values of Hamiltonians, $\hat{H}_{1}^{N}$ and $\hat{H}_{2}^{N}$, where the potentials $v_{1}(\mathbf{r})$ and $v_{2}(\mathbf{r})$ differ by more than a constant, we have,

$$
\begin{aligned}
& E_{1}<\left\langle\Psi_{2}^{K N}\left|\hat{H}_{1}^{N}\right| \Psi_{2}^{K N}\right\rangle \\
= & \left\langle\Psi_{2}^{K N}\left|v_{1}+\hat{T}^{N}+\hat{U}^{N}+v_{2}-v_{2}\right| \Psi_{2}^{K N}\right\rangle \\
= & \left\langle\Psi_{2}^{K N}\left|v_{2}+\hat{T}^{N}+\hat{U}^{N}+v_{1}-v_{2}\right| \Psi_{2}^{K N}\right\rangle \\
= & E_{2}+\int n(\mathbf{r})\left[v_{1}(\mathbf{r})-v_{2}(\mathbf{r})\right] \mathrm{d} \mathbf{r} .
\end{aligned}
$$

Interchanging the indices 1 and 2 in the last expressions, and adding, we obtain the contradiction,

$$
E_{1}+E_{2}<E_{2}+E_{1} .
$$

It follows that there cannot be two potentials that differ by more than a constant leading to the same density for their respective ground states.

From this follows the generalization of the first theorem of Hohenberg and Kohn: the ground-state density of a collection of $N$-particle systems subject to the Pauli principle defines uniquely (modulo a constant) the external potential acting on the system of particles.

It is seen that the non-degenerate ground-state energy of an interacting system (collection of replicas) under an external potential can be written in functional form,

$$
E_{v}[n]=\int v(\mathbf{r}) n(\mathbf{r}) \mathrm{d} \mathbf{r}+\left\langle\Psi_{\mathrm{GS}}^{K N}\left|\hat{T}^{N}+\hat{U}^{N}\right| \Psi_{\mathrm{GS}}^{K N}\right\rangle .
$$


Degeneracy can be treated within the constrained search formalism of Levy and Lieb [36, 37. We now can formulate and prove the generalization of the second theorem of Hohenberg and Kohn to excited states. The language is identical to that in the ground-state formulation [32]: For any trial density, $\tilde{n}(\mathbf{r})$, normalized to $N$, we have,

$$
E_{v}[n] \leq E_{v}[\tilde{n}(\mathbf{r})]
$$

130 state density determines uniquely (modulo a constant) the potential, and hence the Hamiltonian, $\hat{H}^{N}$, of the interacting system characterized by $n(\mathbf{r})$. Let $\tilde{\Psi}^{K N}$ be an anti-symmetric wave function that leads to $\tilde{n}(\mathbf{r}) \neq n(\mathbf{r})$. Because $\tilde{\Psi}^{K N} \neq \Psi_{\mathrm{GS}}^{K N}$, application of the variational principle with $\tilde{\Psi}^{K N}$ playing the 135 role of a trial wave function for $\hat{H}^{N}$ leads to the inequality,

$$
\begin{aligned}
& \left\langle\tilde{\Psi}^{K N}\left|\hat{H}^{N}\right| \tilde{\Psi}^{K N}\right\rangle=\left\langle\tilde{\Psi}^{K N}\left|v+\hat{T}^{N}+\hat{U}^{N}\right| \tilde{\Psi}^{K N}\right\rangle \\
= & \int v(\mathbf{r}) \tilde{n}(\mathbf{r}) \mathrm{d} \mathbf{r}+\left\langle\tilde{\Psi}^{K N}\left|\hat{T}^{N}+\hat{U}^{N}\right| \tilde{\Psi}^{K N}\right\rangle \\
\geq & E_{v}[n] .
\end{aligned}
$$

At this point, there seems to be a discrepancy between this proof and the initial one with respect to the bona-fide ground state of an interacting system. No mention has been made of the functional nature of the quantity, $\left\langle\Psi_{\mathrm{GS}}^{K N}\right| \hat{T}^{N}+$ $\hat{U}^{N}\left|\Psi_{\mathrm{GS}}^{K N}\right\rangle$, or the corresponding one expressed in terms of $\left|\tilde{\Psi}^{K N}\right\rangle$. Indeed, I have been reluctant to even employ the notation, $F_{\mathrm{HK}}[n]$, signifying the so-called Hohenberg and Kohn functional.

My reason is two-fold. First, the notation, $F_{\mathrm{HK}}[n]=\left\langle\Psi_{\mathrm{GS}}^{K N}\left|\hat{T}^{N}+\hat{U}^{N}\right| \Psi_{\mathrm{GS}}^{K N}\right\rangle$, may give rise to the notion that the Hohenberg and Kohn functional, $F_{\mathrm{HK}}[n]$, is a mere function of the density. Second, and related to the above, it raises questions about the differentiability of $F_{\mathrm{HK}}[n]$ with respect to the density.

Starting with the latter, Cioslowski [33, 34, 35] has demonstrated how to construct formally all wave functions that sum up to a given density and shown that all, not only those that are functionals of the density, e.g., correspond to ground states of a Hamiltonian, are differentiable with respect to the density. 
This means that every expectation value of the operator, $\hat{T}^{N}+\hat{U}^{N}$, formed in terms of wave functions that lead to a given density, are differentiable with respect to that density.

From this we obtain through explicit functional differentiation,

$$
\frac{\delta\left\langle\Psi_{\mathrm{GS}}^{K N}\left|\hat{T}^{N}+\hat{U}^{N}\right| \Psi_{\mathrm{GS}}^{K N}\right\rangle}{\delta n(\mathbf{r})}=-v(\mathbf{r})
$$

where the derivative is determined modulo an arbitrary constant. The ability to obtain functional derivatives of expectation values with respect to the density solves the long-standing problem of determining the potential from knowledge of its ground state density, establishing constructively the functional, $(n(\mathbf{r}), v(\mathbf{r}))$, for any density that is $v$-representable with respect to the ground state of a potential.

The constrained search and the existence of differentiability of wave functions leading to the density has been used [38] recently to solve the $v$-representability problem, i.e., given a density, and only that density, provides a formal procedure that can decide as to whether the density represents the ground state of a potential, and in case of an affirmative answer determines the potential (modulo a constant), the wave function of the ground state, and the ground-state energy. The concept of $N$-representability and of the constrained search as well as the solution of the $v$-representability problem are immediately applicable to the present formulation of DFT (and will be presented in other work).

The foregoing discussion specifies the manner in which the functional $E_{v}[n]$ is to be evaluated in terms of an arbitrary density, $\tilde{n}(\mathbf{r})$. Implement the constrained search to determine the wave function that leads to $\tilde{n}(\mathbf{r})$ and minimizes the expectation value of $\hat{T}^{N}+\hat{U}^{N}$. Calling that expectation value, $F[\tilde{n}]$, evidently a functional of the density, and noting that the inequality in $(22)$ is valid for any $\left|\tilde{\Psi}^{K N}\right\rangle \rightarrow \tilde{n}(\mathbf{r}) \neq \tilde{n}(\mathbf{r})$, hence for the one that minimizes $\hat{T}^{N}+\hat{U}^{N}$ at $\tilde{n}(\mathbf{r})$, the second theorem can be written in the form,

$$
E_{v}[\tilde{n}]=\int v(\mathbf{r}) \tilde{n}(\mathbf{r})+F[\tilde{n}] \geq E_{v}[n] .
$$

With $F[\tilde{n}]$ replaced by $F_{\mathrm{HK}}[\tilde{n}]$, the last expression reproduces the initial form 
of the theorem 32 . trum of interacting many particle systems. Thus, it amounts to a new paradigm 
in carrying through with the study of quantum systems that is independent of but equivalent to the Schrödinger equation. This concept will be further developed in future work where the concept of $N$-representability will be exploited with respect to systems of replicas to extend the Kohn-Sham formalism [39] to excited states.

\section{Acknowledgement}

I am grateful to X.-G. Zhang, G. M. Stocks, M. Däne, Patrice Turchi and D. M. Nicholson for many fruitful discussions. This work was performed under the auspices of the U.S. Department of Energy by Lawrence Livermore National Laboratory under Contract DE-AC52-07NA27344.

\section{References}

[1] P. Hohenberg, W. Kohn, Inhomogeneous electron gas, Phys. Rev. 136 (3B) (1964) B864-B871. doi:10.1103/PhysRev.136.B864

[ [2] E. K. U. Gross, L. N. Oliveira, W. Kohn, Rayleigh-ritz variational principle for ensembles of fractionally occupied states, Phys. Rev. A 37 (1988) 28052808. doi:10.1103/PhysRevA.37.2805. URL http://link.aps.org/doi/10.1103/PhysRevA.37.2805

215 [3] E. K. U. Gross, L. N. Oliveira, W. Kohn, Density-functional theory for ensembles of fractionally occupied states. i. basic formalism, Phys. Rev. A 37 (1988) 2809-2820. doi:10.1103/PhysRevA.37.2809 URL http://link.aps.org/doi/10.1103/PhysRevA.37.2809

n [4] L. N. Oliveira, E. K. U. Gross, W. Kohn, Density-functional theory for 220 ensembles of fractionally occupied states. ii. application to the he atom, Phys. Rev. A 37 (1988) 2821-2833. doi:10.1103/PhysRevA.37.2821.

URL http://link.aps.org/doi/10.1103/PhysRevA.37.2821 
[5] J. Katriel, An alternative interpretation of theophilou's extension of the hohenberg-kohn theorem to excited states, Journal of Physics C: Solid State Physics 13 (15) (1980) L375.

URL http://stacks . iop.org/0022-3719/13/i=15/a=001

[ [6] N. Hadjisavvas, A. Theophilou, Rigorous formulation of slater's transition-

1. State theory for excited states, Phys. Rev. A 32 (1985) 720-724. doi: 10.1103/PhysRevA.32.720.

230 URL http://link.aps.org/doi/10.1103/PhysRevA.32.720

q [7] J. P. Perdew, M. Levy, Extrema of the density functional for the energy: Excited states from the ground-state theory, Phys. Rev. B 31 (1985) 62646272. doi:10.1103/PhysRevB.31.6264.

URL http://link.aps.org/doi/10.1103/PhysRevB.31.6264

${ }_{235}^{23}$ [8] L. Fritsche, Generalized kohn-sham theory for electronic excitations in reala istic systems, Phys. Rev. B 33 (1986) 3976-3989. doi:10.1103/PhysRevB. 33.3976 .

URL http://link.aps.org/doi/10.1103/PhysRevB.33.3976

q [9] H. Englisch, H. Fieseler, A. Haufe, Density-functional calculations for 240 excited-state energies, Phys. Rev. A 37 (1988) 4570-4576. doi:10.1103/ PhysRevA.37.4570.

URL http://link . aps.org/doi/10.1103/PhysRevA.37.4570

[10] W. Kohn, Density-functional theory for excited states in a quasi-localdensity approximation, Phys. Rev. A 34 (1986) 737-741. doi:10.1103/ PhysRevA.34.737.

URL http://link .aps .org/doi/10.1103/PhysRevA.34.737

[11] M. Levy, A. Nagy, Variational density-functional theory for an individ1. ual excited state, Phys. Rev. Lett. 83 (1999) 4361-4364. doi:10.1103/ PhysRevLett.83.4361.

URL http://link.aps .org/doi/10.1103/PhysRevLett.83.4361 
[12] A. Görling, Density-functional theory beyond the hohenberg-kohn theorem, Phys. Rev. A 59 (1999) 3359-3374. doi:10.1103/PhysRevA.59.3359.

URL http://link.aps.org/doi/10.1103/PhysRevA.59.3359

[13] A. Nagy, M. Levy, Variational density-functional theory for degenerate excited states, Phys. Rev. A 63 (2001) 052502. doi:10.1103/PhysRevA.63. 052502 .

URL http://link.aps .org/doi/10.1103/PhysRevA.63.052502

[14] L. J. Bartolotti, Time-dependent extension of the hohenberg-kohn-levy ¿ energy-density functional, Phys. Rev. A 24 (1981) 1661-1667. doi:10. 1103/PhysRevA.24.1661.

URL http://link. aps .org/doi/10.1103/PhysRevA.24.1661

[15] L. J. Bartolotti, The hydrodynamic formulation of time-dependent KohnSham orbital density functional theory, The Journal of Physical Chemistry 90 (22) (1986) 5518-5523. arXiv:http://dx.doi.org/10.1021/ j100280a009, doi:10.1021/j100280a009. URL http://dx.doi.org/10.1021/j100280a009

[16] E. Runge, E. K. U. Gross, Density-functional theory for timen dependent systems, Phys. Rev. Lett. 52 (1984) 997-1000. doi:10.1103/ PhysRevLett.52.997. URL http://link .aps .org/doi/10.1103/PhysRevLett.52.997

[17] R. van Leeuwen, Time-Dependent Density Functional Theory, Springer, Berlin, Heidelberg, New York, 2006.

[18] N. I. Gidopoulos, P. G. Papaconstantinou, E. K. U. Gross, Spurious inter1 actions, and their correction, in the ensemble-kohn-sham scheme for excited 275 states, Phys. Rev. Lett. 88 (2002) 033003. doi:10.1103/PhysRevLett. 88. 033003 .

URL http://link .aps .org/doi/10.1103/PhysRevLett.88.033003 
[19] N. Gidopoulos, A. Theophilou, Hartree-fock equations determining the optimum set of spin orbitals for the expansion of excited states, Philosophical Magazine Part B 69 (5) (1994) 1067-1074. arXiv:http://dx.doi.org/ 10.1080/01418639408240176, doi:10.1080/01418639408240176

URL http://dx.doi .org/10.1080/01418639408240176

[20] I. Andrejkovics, A. Nagy, Excitation energies in density functional a theory: comparison of several methods for the $\mathrm{H}_{2} \mathrm{O}, \mathrm{N}_{2}, \mathrm{CO}$ and

285

口

[21] A. Nagy, An alternative optimized potential method for ensembles of exdoi:10.1016/S0009-2614(98)01075-6.

URL http://www.sciencedirect.com/science/article/pii/ S0009261498010756

cited states, Journal of Physics B: Atomic, Molecular and Optical Physics 34 (12) (2001) 2363.

URL http://stacks .iop.org/0953-4075/34/i=12/a=305

[22] A. K. Theophilou, The energy density functional formalism for excited states, Journal of Physics C: Solid State Physics 12 (24) (1979) 5419.

URL http://stacks.iop.org/0022-3719/12/i=24/a=013

[23] N. I. Gidopoulos, P. G. Papaconstantinou, E. K. U. Gross, Physica B 318 (2002) 328.

[24] A. K. Theophilou, P. G. Papaconstantinou, Local spin-density approximation for spin eigenspaces and its application to the excited states of atoms, Phys. Rev. A 61 (2000) 022502. doi:10.1103/PhysRevA.61.022502.

URL http://link .aps.org/doi/10.1103/PhysRevA.61.022502

[25] V. N. Glushkov, A. K. Theophilou, Finite-basis-set implementation of subspace density-functional theory for excited states, Phys. Rev. A 64 (2001) 064501. doi:10.1103/PhysRevA.64.064501.

URL http://link .aps .org/doi/10.1103/PhysRevA.64.064501 $\mathrm{C}_{2} \mathrm{H}_{4}$ molecules, Chemical Physics Letters 296 (5-6) (1998) 489-493. 
[26] J. Katriel, F. Zahariev, Hamiltonian formulation of weighted-ensemble

density-functional theory, Phys. Rev. A 65 (2002) 024501. doi:10.1103/ PhysRevA.65.024501

URL http://link.aps.org/doi/10.1103/PhysRevA.65.024501

310 27] G. Hong, M. Strajbl, T. A. Wesolowski, A. Warshel, Constraining the

a electron densities in DFT method as an effective way for ab initio studies of metal-catalyzed reactions, Vol. 21, John Wiley and Sons, Ltd,

1. 2000, pp. 1554-1561. doi:10.1002/1096-987X(200012)21:16<1554:: AID-JCC12>3.0.CO;2-I.

315 URL http://dx.doi.org/10.1002/1096-987X(200012)21:16<1554:: AID-JCC12>3.0.CD;2-I

[28] V. N. Glushkov, X. Assfeld, Doubly, triply, and multiply excited states from a constrained optimized effective potential method, The Journal of Chemical Physics 132 (20) (2010) 204106. doi:10.1063/1.3443777.

320 a URL http://scitation.aip.org/content/aip/journal/jcp/132/20/ $10.1063 / 1.3443777$

[29] T. A. Johnson, E. Urban, T. Henage, L. Isenhower, D. D. Yavuz, T. G.

n Walker, M. Saffman, Rabi oscillations between ground and rydberg states with dipole-dipole atomic interactions, Phys. Rev. Lett. 100 (2008) 113003.

325 doi:10.1103/PhysRevLett.100.113003.

URL http://link.aps.org/doi/10.1103/PhysRevLett.100.113003

[30] S. Kümmel, L. Kronik, Orbital-dependent density functionals: Theory

口 and applications, Rev. Mod. Phys. 80 (1) (2008) 3-60. doi:10.1103/ RevModPhys.80.3.

[31] B. C. Carlson, J. M. Keller, Eigenvalues of density matrices, Phys. Rev. 121 (1961) 659-661. doi:10.1103/PhysRev.121.659

URL http://link.aps.org/doi/10.1103/PhysRev.121.659

[32] R. G. Parr, C. Y. Yang, Density Functional Theory of Atoms and Molecules, Oxford University Press, Oxford, 1989. 
[33] J. Cioslowski, Density functionals for the energy of electronic systems: Explicit variational construction, Phys. Rev. Lett. 60 (1988) 2141-2143. doi:10.1103/PhysRevLett.60.2141

URL http://link.aps .org/doi/10.1103/PhysRevLett.60.2141

[34] J. Cioslowski, Density driven self-consistent field method. i. derivation and basic properties, The Journal of Chemical Physics 89 (8) (1988) 4871-4874. doi:10.1063/1.455655.

URL http://link.aip.org/link/?JCP/89/4871/1

[35] J. Cioslowski, Density driven self-consistent field method. ii. construction of all one-particle wave functions that are orthonormal and sum up to a given density, International Journal of Quantum Chemistry Quantum Chemistry Symposium 23 (8) (1989) 255-262.

[36] M. Levy, Universal variational functionals of electron densities, firstorder density matrices, and natural spin-orbitals and solution of the $\mathrm{v}$ representability problem, Proceedings of the National Academy of Sciences 76 (12) (1979) 6062-6065. arXiv:http://www.pnas .org/content/76/12/ 6062.full.pdf+html. URL http://www . pnas .org/content/76/12/6062.abstract

[37] E. H. Lieb, Density functionals for coulomb systems, in: A. Shimony, H. Feshbach (Eds.), Physics as Natural Philosophy, Essays in Honor of Laszlo Tisza, MIT Press Cambridge, Massachusetts, 1982, See also, E. H. Lieb, in Density Functional methods in Physics, ed. R. M. Dreizler, NATO ASI Series B123, (Plenum, New York, 1985) p.31, and E. H. Lieb, in Int. J. Quantum Chem. 24, 243 (1983).

[38] A. Gonis, X.-G. Zhang, M. Dne, G. Stocks, D. Nicholson, Re360 formulation of density functional theory for n-representable densities and the resolution of the v-representability problem, Journal of Physics and Chemistry of Solids 89 (2016) $23 \quad-31$. doi:http://dx.doi.org/10.1016/j.jpcs.2015.10.006 
a URL http://www.sciencedirect.com/science/article/pii/

365 S0022369715300743

[39] W. Kohn, L. J. Sham, Self-consistent equations including exchange and correlation effects, Phys. Rev. 140 (4A) (1965) A1133-A1138. doi:10. 1103/PhysRev.140.A1133. 